PROCEEDINGS OF THE

AMERICAN MATHEMATICAL SOCIETY

Volume 126, Number 5, May 1998, Pages 1337-1343

S $0002-9939(98) 04491-8$

\title{
THE PRIMITIVE $p$-FROBENIUS GROUPS
}

\author{
P. FLEISCHMANN, W. LEMPKEN, AND PHAM HUU TIEP \\ (Communicated by Ronald M. Solomon)
}

\begin{abstract}
Let $p$ be a fixed prime. A finite primitive permutation group $G$ with every two-point stabilizer $G_{\alpha, \beta}$ being a $p$-group is called a primitive $p$ Frobenius group. Using our earlier results on $p$-intersection subgroups, we give a complete classification of the primitive $p$-Frobenius groups.
\end{abstract}

\section{INTRODUCTION}

Let $\Sigma_{\Omega}$ denote the symmetric group on a finite set $\Omega$ and $G \leq \Sigma_{\Omega}$ a transitive permutation group. Suppose that every two-point stabilizer $G_{\alpha, \beta}$ is trivial; then a classical result of Frobenius states that $G$ is a semidirect product $K: G_{\alpha}$, where the normal subgroup $K$ consists precisely of the identity together with all elements $g \in G$ that do not fix any point of $\Omega$. Moreover the point stabilizer $G_{\alpha}$ acts on $K$ (via conjugation) in such a way that no nontrivial element $g \in G_{\alpha}$ has a nontrivial fixed point in $K$. A permutation group with these properties is called a Frobenius group with Frobenius kernel $K$.

As a consequence of a celebrated theorem of J. G. Thompson, the Frobenius kernel $K$ is nilpotent and $G_{\alpha}$ is a semiregular group, i.e. $G_{\alpha}$ has a faithful action on a suitable vector space $V$ such that $C_{V}(g)=0$ for every $g \in G_{\alpha} \backslash\{i d\}$ (notice that $V$ can be chosen as a characteristically simple subgroup of a Sylow group of $K)$. If moreover $G$ is primitive, then $K \cong \mathbb{Z}_{p}^{\ell}=: V$ and $G_{\alpha}$ acts semiregularly on $V$.

In the course of his investigation of finite near fields, Zassenhaus [9] obtained a complete classification of finite semiregular groups. Putting all these results together, one has a complete picture of primitive Frobenius groups.

In this paper we finish our investigation of the following $p$-local variant of the situation above:

Definition 1.1. Let $p$ be a fixed prime. We define $\mathcal{F}(p)$ to be the set of all (faithful) finite primitive permutation groups with every 2-point stabilizer $G_{\alpha, \beta}$ being a $p$ group. An element of $\mathcal{F}(p)$ will be called a primitive $p$-Frobenius group.

Furthermore, $\mathcal{F}^{a}(p)$ denotes the subset of $\mathcal{F}(p)$ consisting of those groups with abelian socle, and we set $\mathcal{F}^{n a}(p):=\mathcal{F}(p) \backslash \mathcal{F}^{a}(p)$.

In [3], we defined a proper subgroup $X<G$ to be a p-intersection subgroup of $G$ if and only if $X$ is not a $p$-group but for any $g \in G \backslash X$ the intersection $X \cap X^{g}$ is a $p$-group. The set of $p$-intersection subgroups of $G$ will be denoted by $\mathcal{I}_{p}(G)$. The

Received by the editors June 19, 1996 and, in revised form, November 5, 1996.

1991 Mathematics Subject Classification. Primary 20B15.

(C)1998 American Mathematical Society 
main result of [3] is a classification of the elements in $\mathcal{I}_{p}(G)$ for all almost simple groups. Now we will apply this result to obtain a complete classification of the primitive $p$-Frobenius groups.

Remarks. (i) It is clear that $\mathcal{F}(p)$ includes the class $\mathcal{F}^{\infty}$ of all finite primitive Frobenius groups, which is the intersection $\bigcap_{p \text { prime }} \mathcal{F}(p)$.

(ii) Every finite group $G$ with $O_{p}(G)=1$ admits a faithful transitive permutation representation with every $G_{\alpha, \beta}$ being a $p$-group: take the action of $G$ on $G / P, P \in$ $\operatorname{Syl}_{p}(G)$. Thus it is the primitivity condition that makes the class $\mathcal{F}(p)$ interesting.

(iii) It is immediate from the definition, that $G \in \mathcal{F}(p)$ implies that either $G_{\alpha}$ is a $p$-group or $G_{\alpha} \in \mathcal{I}_{p}(G)$.

Our notation will be as follows: $\Omega$ always denotes a finite set and $G$ a finite group with $\pi(G)$ the set of prime divisors of $|G|$. For $\alpha \in \Omega$ and $G \leq \Sigma_{\Omega}$ we write $G_{\alpha}$ for $\operatorname{Stab}_{G}(\alpha)$. For any subgroup $S \leq G$ we set $A u t_{G}(S):=N_{G}(S) / C_{G}(S)$. Also, $X<\cdot Y$ means that $X$ is a maximal subgroup of a group $Y$. The socle of $G$, $\operatorname{soc}(G)$, is the product of all minimal normal subgroups of $G$. A subgroup $G \leq \Sigma_{\Omega}$ is called primitive (resp. regular) if it is transitive and $G_{\alpha}<G$ (resp. $G_{\alpha}=1$ ). The symmetric, resp. alternating, group on $n$ symbols is denoted by $\Sigma_{n}$, resp. $\mathcal{A}_{n}$.

Observe that if $G \in \mathcal{F}^{a}(p)$ then $\operatorname{soc}(G)=V=\mathbb{F}_{\ell}^{n}$ is elementary abelian (the prime $\ell$ may differ from $p$ ) and $G$ is the semi-direct product of $V$ and any point stabilizer $G_{\alpha}, a \in \Omega$. In this case, one can identify $\Omega$ with $V, a \in \Omega$ with the zero vector of $V$, then embed $G_{0}$ in $G L(V)$ so that the action of $G_{\alpha}=G_{0}$ on $\Omega$ and the linear action of $G_{0}$ on $V$ are compatible.

Let us recall the following definition of [2]: A pair $(G, V)$ consisting of a finite group $G$ and a finite-dimensional $\mathbb{F} G$-module $V$ over some field $\mathbb{F}$ is called $p^{\prime}$ semiregular if every nontrivial $p^{\prime}$-element of $G$ acts without any fixed points on $V \backslash\{0\} . G$ is called $p^{\prime}$-semiregular if $(G, V)$ is $p^{\prime}$-semiregular for a suitable $V$.

Now the following statement is immediate:

Proposition 1.2. Suppose $\operatorname{soc}(G)=V$ is elementary abelian. Then $G \in \mathcal{F}^{a}(p)$ if and only if the pair $\left(G_{0}, V\right)$ is $p^{\prime}$-semiregular, and $V$ is a faithful irreducible $G_{0}$-module. ○

In [2] all $p^{\prime}$-semiregular pairs $\left(G_{0}, V\right)$ have been determined.

The present paper provides a proof of the following complete classification of the groups in $\mathcal{F}(p)$ :

Theorem 1.3. Let $G$ be an element of $\mathcal{F}(p)$ and put $S:=\operatorname{soc}(G)$. Then precisely one of the following three cases occurs:

(i) $G$ has a regular normal subgroup $V$. In this case $V=S$ is elementary abelian, $G \in \mathcal{F}^{a}(p)$ and $G=V: G_{0}$ with $p^{\prime}$-semiregular pair $\left(G_{0}, V\right)$ as described in [2] (and $V$ is a faithful irreducible $G_{0}$-module).

(ii) $G$ has no regular normal subgroup and $G_{\alpha}$ is nilpotent. In this case $p=2$, $G \in \mathcal{F}^{n a}(2), G_{\alpha} \in \operatorname{Syl}_{2}(G)$ and $S=O^{2}(G)=S_{1} \times \cdots \times S_{k}$ is the unique minimal normal subgroup of $G$; moreover, $S_{1} \cong \cdots \cong S_{k} \cong L_{2}(q)$ with $q=2^{n} \pm 1>5$ a prime or $q=9$. Furthermore, $[G: S]$ and $k$ are powers of 2 .

(iii) $G$ has no regular normal subgroup and a point stabilizer $G_{\alpha}$ is an element of $\mathcal{I}_{p}(G)$. In this case $S$ is simple, i.e. $G$ is almost simple. Furthermore, the tuples $\left(S, G, p, G_{\alpha}\right)$ are as listed in Table I below. 
TABLE I. $p$-intersection maximal subgroups in almost simple groups

\begin{tabular}{|c|c|c|c|}
\hline$S$ & $G$ & $p$ & $G_{\alpha}$ \\
\hline$\overline{\mathcal{A}_{5}}$ & $G=S$ & 3 & $\mathcal{A}_{4}$ \\
\hline $\mathcal{A}_{5}$ & $\mathcal{A}_{5} \leq G \leq \Sigma_{5}$ & 2 & $N_{G}\left(\mathbb{Z}_{3}\right)$ \\
\hline $\mathcal{A}_{6}$ & $\begin{array}{l}\mathcal{A}_{6} \leq G \\
\leq \operatorname{Aut}\left(\mathcal{A}_{6}\right)\end{array}$ & 2 & $N_{G}\left(3^{2}\right)$ \\
\hline $\mathcal{A}_{6}$ & $\begin{array}{l}P G L_{2}(9), M_{10} \\
A u t\left(\mathcal{A}_{6}\right)\end{array}$ & 2 & $N_{G}\left(\mathbb{Z}_{5}\right)$ \\
\hline $\mathcal{A}_{n}, n$ prime $\notin\{7,11,17,23\}$ & $G=S$ & $p$ with $\frac{n-1}{2}=p^{f}$ & $\mathbb{Z}_{n}: \mathbb{Z}_{\frac{n-1}{2}}$ \\
\hline $\mathcal{A}_{n}, 5 \leq n \in \mathcal{F}$ & $\Sigma_{n}$ & 2 & $\mathbb{Z}_{n}: \mathbb{Z}_{n-1}$ \\
\hline $\begin{array}{l}L_{2}(7) \cong L_{3}(2) \\
L_{2}(7)\end{array}$ & $\begin{array}{l}G=S \\
G=P G L_{2}(7) \\
G=S\end{array}$ & $\begin{array}{l}2 \\
2 \\
3\end{array}$ & $\begin{array}{l}\Sigma_{4}^{(i)}, i=1,2 \\
\mathcal{N}(X), N\left(T_{1}\right) \\
\quad \cong D_{12} \\
B\end{array}$ \\
\hline$L_{2}(11)$ & $\begin{array}{l}G=S \\
P G L_{2}(11)\end{array}$ & $\begin{array}{l}2 \\
2\end{array}$ & $\begin{array}{l}N\left(T_{\operatorname{cox}}\right) \\
N\left(T_{1}\right) \cong D_{20} \\
N\left(T_{\text {cox }}\right) \cong D_{24}\end{array}$ \\
\hline$L_{2}(11)$ & $G=S$ & 5 & $B$ \\
\hline$L_{2}\left(2^{a}\right), 2^{a} \geq 4$ & $G=S$ & $p=2^{a}-1 \in \mathcal{M}$ & $B$ \\
\hline$L_{2}(q), q \notin \overline{\mathcal{F}} \cup\{4,7,9,11\}$ & $\pi(G / S) \subseteq\{2\}$ & 2 & $N\left(T_{1}\right)$ \\
\hline$L_{2}(q), q \notin \mathcal{M} \cup\{4,5,9,11\}$ & $\pi(G / S) \subseteq\{2\}$ & 2 & $N\left(T_{\operatorname{cox}}\right)$ \\
\hline$L_{2}\left(2^{\ell}\right)$ & $\begin{aligned} 2 & <|G / S| \\
& =\ell \text { prime }\end{aligned}$ & 2 & $N\left(T_{\operatorname{cox}}\right)$ \\
\hline$L_{2}(r), r=2 p^{x}+1$ a prime & $G=S$ & $p>2$ & $B$ \\
\hline$L_{2}\left(3^{m}\right),\left\{\begin{array}{l}3^{m}=2 p^{x}+1 \\
m \mid(p-1) \\
m \text { an odd prime }\end{array}\right.$ & $G=S$ & $p>2$ & $B$ \\
\hline$L_{2}(r), r \in \mathcal{F}$ & $G / S \leq \mathbb{Z}_{2}$ & 2 & $B$ \\
\hline$P S U_{3}(q), q \in\{3,5,9\}$ & $G / S \leq \mathbb{Z}_{2}$ & 2 & $B$ \\
\hline${ }^{2} G_{2}(3)^{\prime} \cong L_{2}(8)$ & $G / S \leq \mathbb{Z}_{3}$ & 2 & $B, N_{G}(B)$ \\
\hline${ }^{2} B_{2}(q), q=2^{l}, l=2 a+1>1$ & $\begin{array}{l}G=S \\
G=S \\
|G / S|=l \text { prime }\end{array}$ & $\begin{array}{c}p=2^{l}-1 \in \mathcal{M} \\
2 \\
2\end{array}$ & $\begin{array}{l}B \\
D_{2(q-1)} \\
X_{ \pm}=\mathbb{Z}_{q \pm 2 \sqrt{q}+1}: \mathbb{Z}_{4} \\
X_{ \pm}: \mathbb{Z}_{l} \text { with } 5 \| X_{ \pm} \mid\end{array}$ \\
\hline$L_{3}(4)$ & $G / S \leq F \cong \mathbb{Z}_{2}$ & 2 & $N\left(\mathbb{Z}_{3}^{2}: Q_{8}\right)$ \\
\hline$L_{3}(4)$ & $\begin{array}{l}\Delta \leq \bar{S} / S \\
\leq \mathbb{Z}_{2} \times \mathbb{Z}_{2}\end{array}$ & 2 & $N\left(\mathbb{Z}_{3}^{2}: Q_{8}\right)$ \\
\hline${ }^{3} D_{4}(q)$ & $\pi(G / S) \subseteq\{2\}$ & 2 & $\begin{array}{l}N\left(T_{5}\right) \\
\quad\left|T_{5}\right|=q^{4}-q^{2}+1\end{array}$ \\
\hline$L_{p}(q), P S U_{p}(q)$ & $\pi(G / S) \subseteq\{p\}$ & $p$ & $N\left(T_{\operatorname{cox}}\right)$ \\
\hline 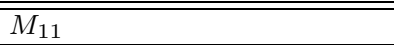 & $\overline{G=S}$ & 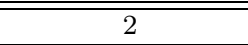 & $\overline{3^{2}: Q_{8} \cdot 2}$ \\
\hline$J_{1}$ & $G=S$ & 2 & $\Sigma_{3} \times D_{10}$ \\
\hline$M_{23}$ & $G=S$ & 11 & $23: 11$ \\
\hline$B M$ & $G=S$ & 23 & $47: 23$ \\
\hline$M(? ?)$ & $G=S$ & 29 & $59: 29$ \\
\hline
\end{tabular}

Conversely, each of the groups mentioned in (i), (ii) and (iii) is a member of $\mathcal{F}(p)$, where in case (ii) we have to assume in addition that $\operatorname{Aut}_{G}\left(S_{i}\right) \cong P G L_{2}(7)$ if $q=7$ and $\operatorname{Aut}_{G}\left(S_{i}\right) \cong P G L_{2}(9), M_{10}$ or $\operatorname{Aut}\left(\mathcal{A}_{6}\right)$ if $q=9$.

In Table I $B$ denotes a Borel subgroup; $T_{1}, T_{\text {cox }}$ denote split- and Coxeter tori, respectively, $L_{2}(q):=P S L_{2}(q), N(H):=N_{G}(H)$; if $G>S, \mathcal{N}(X):=$ $\left\{N_{G}(X) \mid X \in \mathcal{I}_{p}(S)\right.$, maximal in $\left.S\right\}$. Here ?? means either $59: 29<L_{2}(59)<\cdot M$ 
or $59: 29<\cdot M$. The existence of $L_{2}(59)$ in $M$ is not settled yet. $\mathcal{F}$, resp. $\mathcal{M}$, denotes the set of Fermat, resp. Mersenne, primes.

Due to the isomorphisms $\mathcal{A}_{5} \cong L_{2}(4) \cong L_{2}(5)$ and $\mathcal{A}_{6} \cong L_{2}(9)$ these groups and their automorphic decorations are listed only in the alternating groups' section. For $L_{3}(4), F$ (resp. $\Delta \cong \mathbb{Z}_{2}$ ) is generated by field (resp. graph) automorphisms.

Note that for any $X \in \mathcal{I}_{p}(G)$ there is a subgroup $H \leq G$ such that $X \in \mathcal{I}_{p}(H)$ and $X$ is maximal in $H$. Hence the results above give also a complete classification of $p$-intersection subgroups occuring in arbitrary finite groups. In particular we obtain the following result which can be viewed as the $p$-local version of Zassenhaus' classification of Frobenius complements:

Theorem 1.4. Let $G$ be a finite group, $p$ a prime and $X<G$ a p-intersection subgroup. Then either $X$ is $p^{\prime}$-semiregular or $X$ is a solvable group (occurring as $G_{\alpha}$ in Table I up to a suitable normal p-subgroup of X).

\section{Prerequisites}

The reader is referred to [2] for a complete list of $p^{\prime}$-semiregular groups; here we restrict ourselves to listing the perfect ones. $\mathcal{R}$ is the set of all primes $r$ such that $r=2^{a} \cdot 3^{b}+1$ for $a \geq 2, b \geq 0$, and $(r+1) / 2$ is a prime.

R. Guralnick and R. Wiegand [5] also classified $p^{\prime}$-semiregular groups $G$ such that the underlying field of the corresponding $G$-modules has characteristic $p$, and pointed out a very interesting connection of these groups with multiplicative structures of Galois field extensions. The authors' proof in [2] is independent of that in [5] and in addition also describes the relevant $G$-modules.

Theorem 2.1. Let $G$ be a perfect finite group and $(G, V)$ a $p^{\prime}$-semiregular pair for a faithful irreducible $\mathbb{F} G$-module $V$. Then one of the following holds:

(i) $G \cong S L_{2}\left(p^{a}\right)$ for some $a \geq 1$ with $p^{a}>3$.

(ii) $G \cong{ }^{2} B_{2}\left(2^{2 a+1}\right)$ for some $a \geq 1$ with $p=2$.

(iii) $G \cong{ }^{2} B_{2}\left(2^{2 a+1}\right) \times S L_{2}\left(2^{2 b+1}\right)$ with $a, b \geq 1, \operatorname{gcd}(2 a+1,2 b+1)=1$ and $p=2$.

(iv) $G \cong S L_{2}(r)$ with $r \in \mathcal{R} \cup\{7,17\}$ and $p=3$.

(v) $G \cong S L_{2}(5)$ and $p \geq 7$.

(vi) $G=E S$, where $E=O_{2}(G) \cong 2_{-}^{1+4}, S \cong S L_{2}(5), E \cap S=Z(G) \cong \mathbb{Z}_{2}$ and $p=2$.

Conversely, if $(G, p)$ satisfies any of the conditions (i) - (vi), then there exists a faithful absolutely irreducible $G$-module $V$ such that $(G, V)$ is $p^{\prime}$-semiregular.

Proof. Let $(G, V)$ be $p^{\prime}$-semiregular for a faithful irreducible $\mathbb{F} G$-module $V$ and $\operatorname{char} \mathbb{F}=\ell$. First suppose that $\ell$ divides $|G|$. Then it is clear that $\ell=p$ and the irreducibility of $V$ forces $O_{p}(G)=1$. By Theorem 4.1 of [2] (cf. also [5]), $(G, p)$ is as listed in (i) - (iv). Next suppose that $\ell$ does not divide $|G|$. Then Theorem 5.6 of [2] and the irreducibility of $V$ force $(G, p)$ to satisfy one of the conditions (iv) (vi) or (i) with $p^{a}=4,5,9$. The existence of $p^{\prime}$-semiregular pairs for the groups $G$ listed has also been established in [2]. $\circ$

Example 2.2. (i) (Zassenhaus) $\ell^{2}: S L_{2}(5) \in \mathcal{F}^{\infty}$ for all primes $\ell \equiv \pm 1(\bmod 10)$ and $\ell^{4}: S L_{2}(5) \in \mathcal{F}^{\infty}$ for all primes $\ell \equiv \pm 3(\bmod 10)$.

(ii) $p^{2 a}: S L_{2}\left(p^{a}\right) \in \mathcal{F}(p)$ for any prime $p$.

(iii) $3^{6}: S L_{2}(13) \in \mathcal{F}(3)$ (Hering's group).

(iv) $7^{8}:\left(2_{-}^{1+4} \backslash \mathcal{A}_{5}\right) \in \mathcal{F}(2)$ and $7^{4}: S L_{2}(9), 5^{12}: S L_{2}(13) \in \mathcal{F}(3)$. 
The following result will be used in the next section.

Theorem 2.3. Let $G$ be a finite group with a nilpotent maximal subgroup $H$.

(i) (Thompson; see [4], Thm. 10.3.2) If $H$ has odd order then $G$ is solvable.

(ii) (Baumann; see [1]) If $G$ is non-solvable then $O^{2}(G / F(G))$ is a direct product of simple groups isomorphic to $L_{2}(q)$ with primes $q$ of the form $2^{n} \pm 1$ or $q=9$.

We will also need the following result, which is an easy consequence of the classification of finite simple groups:

Lemma 2.4. Let $\mathcal{E}$ be a nonabelian finite simple group and let $\alpha \in \operatorname{Aut}(\mathcal{E})$ be an element whose order is coprime to $|\mathcal{E}|$. Then $C_{\mathcal{E}}(\alpha)$ is not nilpotent.

Proof. See [3]. ○

\section{Reduction to the Simple Socle CASE}

Proposition 3.1. Let $G \in \mathcal{F}^{n a}(p)$. Then $G$ does not contain any regular normal subgroup. In particular, $S:=\operatorname{soc}(G)$ is the unique minimal normal subgroup of $G$, $C_{G}(S)=1$, and $G / S \cong G_{\alpha} / S_{\alpha}$.

Moreover, for any point stabilizer $G_{\alpha}$ one of the following is true:

(i) $p=2$ and $G_{\alpha} \in \operatorname{Syl}_{2}(G)$;

(ii) $G_{\alpha} \in \mathcal{I}_{p}(G)$.

Proof. 1) Suppose first that $G_{\alpha}$ is a $p$-group. As $G_{\alpha}$ is maximal and nilpotent, 2.3 implies $p=2$ and so $G_{\alpha} \in \operatorname{Syl}_{2}(G)$.

2 ) Suppose next that $1 \neq R \triangleleft G$ with $R \cap G_{\alpha}=1$. We can assume $R \leq \operatorname{soc}(G)$ and $R$ is not solvable (since $G \in \mathcal{F}^{n a}(p)$ ). Suppose in addition that $G_{\alpha}$ is not a $p$-group. Then we can find $x \in G_{\alpha}$ of prime order $q \neq p$. Now for any $1 \neq y \in R, G_{\alpha} \cap G_{y(\alpha)}$ is a $p$-group. In particular, $x^{y} \notin G_{\alpha}$, so $y^{x} \neq y$ and $x$ acts fixed-point-freely on $R \backslash\{1\}$. By a well-known theorem of Thompson (cf. [4], Thm. 10.2.1) $R$ must be nilpotent, a contradiction. Thus $G_{\alpha}$ must be a $p$-group. By 1$), G_{\alpha} \in S y l_{2}(G)$, and $|R|=\left[G: G_{\alpha}\right]$ is odd. So $R$ (and $G=R \cdot G_{\alpha}$ ) is solvable, again a contradiction.

3) The claims concerning $S$ now follow immediately. Furthermore, if $G_{\alpha}$ is a $p$-group, then (i) is fulfilled due to 1); otherwise one arrives at (ii). $\circ$

Corollary 3.2. Let $G \in \mathcal{F}(p)$. Then $G \in \mathcal{F}^{a}(p)$ if and only if $G$ contains a regular normal subgroup. $\circ$

Proposition 3.3. Suppose that $G \in \mathcal{F}^{n a}(p)$ and $\operatorname{soc}(G)$ is not simple. Then conclusion (ii) of Theorem 1.3 holds.

Proof. A basic tool for studying finite permutation groups is the reduction theorem first stated by O'Nan and Scott (see [8]). Here we are using an expanded version of this theorem given in [7]. Because of 3.2, the primitive permutation group $G$ under question has no regular normal subgroups (and $\operatorname{soc}(G)$ is not simple). In this case, the O'Nan-Scott theorem says that $G$ is either a simple diagonal action or a product action group; cf. [7]. We shall use the notation given there. In particular, $B=\operatorname{soc}(G)=S_{1} \times \ldots \times S_{k}$ with $S_{1} \cong \ldots \cong S_{k} \cong T$ for a non-abelian finite simple group $T$.

1) Suppose $G$ is a simple diagonal action group, that is, case III(a) of [7] occurs. Then for some $\alpha \in \Omega$ one has $B_{\alpha}=\{(a, a, \ldots, a) \mid a \in T\}$. In particular, taking $g:=(a, a, \ldots, a), h:=(a, 1, \ldots, 1)$ for a non-identity $p^{\prime}$-element $a \in T$, one sees 
that $g=g^{h} \in B_{\alpha} \cap B_{h(\alpha)}$. Meanwhile $h \notin B_{\alpha}$, contrary to the assumption that $G \in \mathcal{F}(p)$.

We have shown that $G$ is a product action group, i.e., case III(b) of [7] occurs. In this case, $\Omega$ can be identified with $\Gamma^{\ell}$ for some finite set $\Gamma$ and some $\ell$ dividing $k$. Furthermore, if $H$ denotes $A u t_{G}\left(S_{1} \times S_{2} \times \ldots \times S_{k / \ell}\right)$ (after suitably reindexing the $S_{i}$ 's if necessary), then $H$ acts primitively on $\Gamma$, with socle $K$ isomorphic to $T^{k / \ell}$. Moreover, $H$ is of type II or III(a) (in the notation of [7]). Finally, $G$ can be embedded in $W=H \nmid \Sigma_{\ell}$, and the action of $G$ on $\Omega$ is induced by the natural product action of $W$ on $\Omega$ (cf. [7]).

2) Next we consider the situation when $H$ is of type $\operatorname{III}(\mathrm{a})$. Then $\ell<k$. Arguing as in 1), one sees that $K_{\gamma} \cap K_{\gamma^{\prime}}$ is not a $p$-group for some $\gamma, \gamma^{\prime} \in \Gamma, \gamma \neq \gamma^{\prime}$. For the distinct points $\alpha:=(\gamma, \ldots, \gamma), \alpha^{\prime}:=\left(\gamma^{\prime}, \ldots, \gamma^{\prime}\right)$ in $\Omega$, one has $B_{\alpha}=\left(K_{\gamma}\right)^{\ell}$, $B_{\alpha^{\prime}}=\left(K_{\gamma^{\prime}}\right)^{\ell}$. In particular, $B_{\alpha} \cap B_{\alpha^{\prime}}$ is not a $p$-group, a contradiction.

Thus $H$ must be of type II, i.e., $k=\ell$.

3) At this point we show that $H_{\gamma}$ is a $p$-group.

First observe that $B_{\alpha}$ is a $p$-group, with $\alpha=(\gamma, \ldots, \gamma)$. Indeed, suppose $s=$ $\left(s_{1}, \ldots, s_{k}\right) \in B_{\alpha}$ is not a $p$-element. Without loss we may suppose $s_{1} \in T$ is not a $p$-element. Then $s=s^{t} \in B_{\alpha} \cap B_{t(\alpha)}$ with $t:=\left(s_{1}, 1, \ldots, 1\right)$. This implies that $t \in B_{\alpha}$. Now taking $u:=\left(1, g_{2}, \ldots, g_{k}\right)$, one has $t=t^{u} \in B_{\alpha} \cap B_{u(\alpha)}$ for any $g_{i} \in T$. We conclude that all $\left(1, g_{2}, \ldots, g_{k}\right)$ are contained in $B_{\alpha}$, contradicting the equality $B_{\alpha}=\left(K_{\gamma}\right)^{k}$.

Suppose there is an element $x \in H_{\gamma}$ whose order is a prime $r$ different from $p$. Every element in $W$ can be canonically written in the form $\left(g_{1}, \ldots, g_{k}\right) \pi$ for $g_{i} \in \Sigma_{\Gamma}$ and $\pi \in \Sigma_{k}$. Then $G_{\alpha}$ contains an element $g=\left(x, g_{2}, \ldots, g_{k}\right) \pi$ with $\pi(1)=1$. For any $y \in C_{K}(x)$ and $h:=(y, 1, \ldots, 1)$, we have $g=g^{h} \in G_{\alpha} \cap G_{h(\alpha)}$, yielding $h \in B_{\alpha}$. Due to the above observation $C_{K}(x)$ is a $p$-group. Choose $Q \in \operatorname{Syl}_{r}(H)$ with $x \in Q$. Then $Q \cap K \triangleleft Q$ and $Z(Q) \cap(Q \cap K) \leq C_{K}(x)$. So $1=Z(Q) \cap(Q \cap K)$, which implies that $1=Q \cap K$. But $Q \cap K \in \operatorname{Syl}_{r}(K)$, hence $(r,|K|)=1$. Now 2.4 applied to $K$ and $x$ provides a contradiction.

4) We have proved that $H_{\gamma}$ is a $p$-group. If $p$ is odd, then the maximality of $H_{\gamma}$ in $H$ together with 2.3 implies that $H$ is solvable, a contradiction. So we conclude that $p=2$. We claim that $G_{\alpha}$ is a 2 -group. For, suppose that $g=\left(g_{1}, \ldots, g_{k}\right) \pi \in G_{\alpha}$ has order $r$, an odd prime, and $\alpha=(\gamma, \ldots, \gamma)$. Observe that $G_{\alpha} \leq W_{\alpha}=H_{\gamma} \imath \Sigma_{k}$. Since $g_{i} \in H_{\gamma}$ has order a power of 2 , we conclude that $\pi$ has order $r$. In particular, we may suppose that $\pi$ permutes the groups $S_{1}, \ldots, S_{r}$ cyclically. Choose $c \in K \backslash K_{\gamma}$. Then for $y:=(c, 1, \ldots, 1) \in B$ we have $\left[y^{g^{i}}, y^{g^{j}}\right]=1$ for all $i, j=1,2, \ldots, r$. Hence $\tilde{y}:=y y^{g} y^{g^{2}} \ldots y^{g^{r-1}} \in C_{B}(g)$. From this it follows that $g=g^{\tilde{y}} \in G_{\alpha} \cap G_{\tilde{y}(\alpha)}$, yielding $\tilde{y} \in B_{\alpha}$. But in this case $c$ belongs to $K_{\gamma}$, contrary to the choice of $c$. Consequently, $G_{\alpha}$ is a 2-group, and so $G_{\alpha} \in \operatorname{Syl}_{2}(G)$.

Applying 2.3 to the maximal subgroup $G_{\alpha}$ of $G$, we come to conclusion (ii) of Theorem 1.3. $\circ$

The following is one of the main results in [3]; here it classifies the elements in $\mathcal{F}^{n a}(p)$ with simple socle:

Theorem 3.4. Let $S \unlhd G \leq A u t(S)$ with $S$ a finite nonabelian simple group, and suppose that $X \in \mathcal{I}_{p}(G)$ is maximal in $G$. Then $\left(S, G, p, X=G_{\alpha}\right)$ is one of the tuples listed in Table $I$.

Proof. See [3]. ○ 


\section{Proof of Theorem 1.3}

By 3.2 and the remarks in the introduction we can assume that $G \in \mathcal{F}^{n a}(p)$.

First we suppose that $G_{\alpha}$ is nilpotent. Applying 2.3, we see that $F(G)=1$ (as $\left.G \in \mathcal{F}^{n a}(p)\right)$ and $S:=\operatorname{soc}(G)=O^{2}(G)$ is the direct product of $k$ isomorphic (since $S$ is characteristically simple) simple groups $S_{1} \cong \ldots \cong S_{k} \cong L_{2}(q)$ with $q=2^{n} \pm 1$ a prime or $q=9$. If $k=1$, then a direct inspection of maximal subgroups of $G$ with $L_{2}(q) \leq G \leq \operatorname{Aut}\left(L_{2}(q)\right)$ shows that $p=2$ and $G_{\alpha} \in S y l_{2}(G)$, that is, conclusion (ii) of 1.3 holds. If $k>1$, then, applying 3.3 , one again obtains that $p=2$ and $G_{\alpha} \in S y l_{2}(G)$. Moreover, due to $3.1, G / S \cong G_{\alpha} / S_{\alpha}$ and hence $[G: S]$ is a 2-power. But $G / S$ acts transitively on the set $\left\{S_{1}, \ldots, S_{k}\right\}$; therefore $k$ is a 2-power. Thus (ii) is fulfilled.

Now suppose that (ii) does not hold. Then $G_{\alpha} \in \mathcal{I}_{p}(G)$. In this case 3.3 shows that $\operatorname{soc}(G)$ is simple. Applying 3.4, we arrive at (iii). 。

\section{ACKNOWLEDGEMENTS}

The authors are grateful to Prof. R. Solomon for helpful discussions, and to the referee for his valuable comments that greatly improved the exposition of the paper.

\section{REFERENCES}

[1] B. Baumann, Endliche nichtauflösbare Gruppen mit einer nilpotenten maximalen Untergruppe, J. Algebra 38 (1976), 119 - 135. MR 53:13391

[2] P. Fleischmann, W. Lempken and P.H. Tiep, Finite $p^{\prime}$-semiregular groups, J. Algebra 188 (1997), 547 - 579. CMP 97:08

[3] P. Fleischmann, W. Lempken and P.H. Tiep, The $p$-intersection subgroups in quasi-simple and almost simple finite groups, submitted.

[4] D. Gorenstein, "Finite Groups", Harper and Row, 1968. MR 38:229

[5] R. Guralnick and R. Wiegand, Galois groups and the multiplicative structure of field extensions, Trans. Amer. Math. Soc. 331 (1992), 563 - 584. MR 93c:12004

[6] B. Huppert, "Endliche Gruppen", Springer, 1967. MR 37:302

[7] M. Liebeck, C. Praeger and J. Saxl, On the O'Nan-Scott theorem for finite primitive permutation groups, J. Austral. Math. Soc. (A) 44 (1988), 389 - 396. MR 89a:20002

[8] L. L. Scott, Representations in characteristic p, Santa Cruz Conference on Finite Groups, pp. 318 - 331, Proc. Sympos. Pure Math., vol. 37, Amer. Math. Soc., Providence, R.I., 1980. MR 82e:20052

[9] H. Zassenhaus, Über endliche Fastkörper, Abhandl. Math. Sem. Hamburg. Univ. 11 (1936), $187-220$.

(P. Fleischmann and W. Lempken) Institute for Experimental Mathematics, University of Essen, Ellernstr. 29, 45326 Essen, Germany

(Pham Huu Tiep) Department of Mathematics, Ohio State University, Columbus, Ohio 43210

E-mail address: tiep@math.ohio-state.edu 